\title{
Leprosy elimination through integrated basic health services in Myanmar: the role of midwives
}

\author{
S. BARUA*, S. WAKAI $\dagger$, T. SHWE $\ddagger \&$ T. UMENAI* \\ *Department of Health Policy and Planning, Graduate School of \\ International Health, Faculty of Medicine, University of Tokyo, \\ 7-3-1 Hongo, Bunkyo Ku Tokyo, 113-0033, Japan \\ $\dagger$ Department of Neurosurgery, Dokkyo University School of \\ Medicine, 880 Mibu, Tochigi, 321-02, Japan \\ $\ddagger$ National Leprosy Elimination Program, Department of Health, \\ 36, Theinbyu Road, Yangon, Myanmar
}

\begin{abstract}
Summary Myanmar is one of the top 16 countries identified by WHO as being hyperendemic for leprosy. Multi-drug therapy (MDT) was introduced in 1988 as a vertical programme and gradually integrated into the basic health services (BHS), achieving $100 \%$ coverage over the registered cases by 1995 . To achieve maximum coverage of and benefit for patients, both leprosy vertical staff and BHS staff were trained to implement MDT whilst performing routine BHS activities. This included a total of 8615 trained midwives who were mobilized for the nationwide leprosy elimination programme (LEP). They worked at village level in various parts of the country and were willing and able to carry out basic tasks in leprosy management, such as the implementation of MDT using blister-calender packs carrying a month's supply of drugs. This study was performed to assess the workload of midwives and their attitude towards LEP. The authors conclude that midwives in Myanmar show a high level of commitment and reliability, which are essential contributing factors to achieve the current goal of leprosy elimination by the year 2000. Along with the present trend of decreasing prevalence rate, leprosy could no longer be considered as a public health problem at national level by the year 2000 in Myanmar. However, because of its long incubation period, new leprosy patients may arise even after the elimination target is achieved, whilst many other patients may become disabled. A community-based sustainable approach for the post-elimination phase, after the year 2000 , will be essential and the contribution of the midwives may be of considerable importance.
\end{abstract}




\section{Introduction}

Since the introduction of multi-drug therapy (MDT) in 1982 by the World Health Organization (WHO), ${ }^{1}$ more than 8 million patients have been cured at global level with this treatment. Within 10 years since its introduction, MDT has been adopted by all endemic countries as the standard treatment for leprosy. ${ }^{2}$ MDT is provided in monthly-basis blister packs, making the distribution system easier at different levels of health care services with less workload in the part of the community health workers.,

Myanmar (population 46 million) has a significant leprosy problem and is included by WHO in a list of the top 16 endemic countries worldwide. ${ }^{5,6}$ MDT was introduced in Myanmar in 1988 as a vertical leprosy elimination programme (LEP), which was gradually integrated into the basic health services (BHS) from 1991 and covered the whole country by 1995 (Department of Health, National Leprosy Elimination Programme, Yangon, Myanmar, unpublished Government Report, 1995). At the village level, actual implementation of LEP activities is performed by the Midwives and the Public Health Supervisors II. All the midwives (8615 in 1993-94) nationwide received training on LEP. They gradually adopted LEP in their responsible areas in the integrated BHS services by 1995 (Department of Health, Basic Health Services, Unpublished Government Document, Yangon, Myanmar, 1995). LEP activities such as case finding, confirmation of diagnosis, drug distribution and follow-up of patients became feasible for the midwives and could be performed in conjunction with other BHS activities. The registered prevalence rate of leprosy in Myanmar was reduced from 39.9 in 1988 to 2.91 per 10,000 population in 1997 , whilst the MDT coverage over the registered cases was sharply increased from less than $20 \%$ in 1988 to $100 \%$ in 1995 (Figure 1). This achievement was made through the combined effort of both vertical (specialized) leprosy and

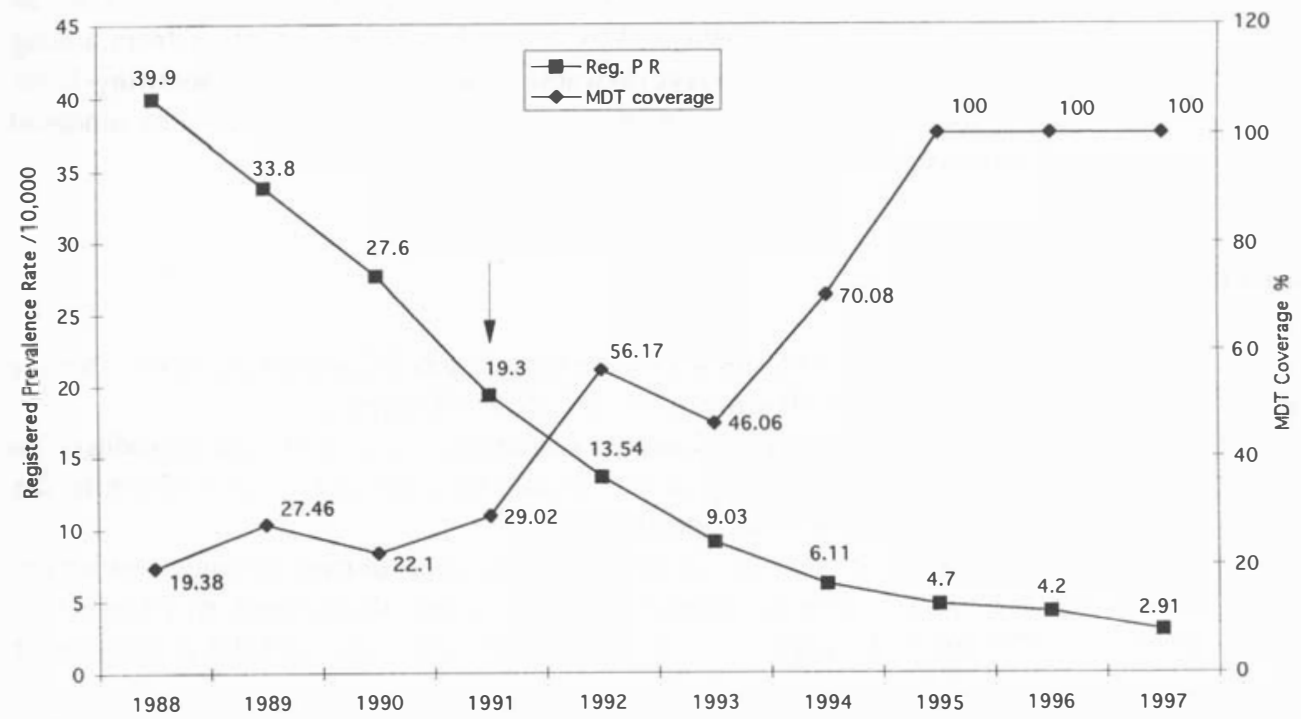

Figure 1. Registered prevalence rate (Reg. P R) and multi-drug therapy (MDT) coverage rate of leprosy cases in Myanmar, 1988-1997. Arrow indicates the time (year) of the integration of the vertical leprosy control activities into BHS and intensive training of BHS staff on leprosy. Source: National Leprosy Elimination Program, Myanmar, 1998. 
BHS staff with midwives playing a vital role (Tin Shwe: Progress Towards Leprosy Elimination in Myanmar; paper presented at the joint Department of Health/World Health Organization meeting inaugurating an Independent Evaluation of the National Leprosy Elimination Programme, Yangon, Myanmar, 4 November, 1997).

Significant contributions of midwives in leprosy elimination activities of Myanmar ${ }^{7}$ are mentioned in several WHO reports that evaluated the overall LEP national programme of Myanmar (Department of Health, Myanmar, final report of the joint Government/WHO Independent Evaluation of Leprosy Control Program, Myanmar, 1993. Unpublished Government Report, Feenstra P. Leprosy Control in Myanmar; Expanding MDT Coverage, Unpublished WHO assignment report, Yangon, Myanmar, 1993).

However, a detailed assessment of the role of the midwives has not been done in terms of performing the LEP activities. This study was thus carried out to analyse the leprosy situation in Myanmar over time: 1) to investigate the role and contribution of the midwives in LEP; 2) to analyse the perceptions of the midwives regarding leprosy activities. We also discuss their possible future contribution to leprosy control beyond the year 2000 in Myanmar.

\section{Subjects and methods}

In Myanmar, under the integrated LEP, three categories of BHS staff are considered as implementers, namely, midwives, public health supervisor grade II and multipurpose health workers, of whom the majorities are midwives.

In order to analyse the LEP workload of the midwives, a survey was conducted by trained interviewers by using pretested standard questionnaire from July to November, 1995 in three of the six Divisions of Myanmar where MDT was introduced in 1988. A total of 188 midwives were interviewed. They were selected by a simple random sampling from among the midwives assigned in three divisions (Ayeyarwaddy, Bago and Yangon), totalling 2349.

The study did not include any analysis of the quality of the leprosy services rendered by the interviewed midwives.

\section{Results}

Of the 188 midwives interviewed, $185(98.4 \%)$ were female and $3(1.6 \%)$ were male. The age ranged from 22 to 59 years old, with a mean of 41.2 (SD \pm 9.4 years).

One hundred and eighteen $(62.8 \%)$ midwives responded that MDT was introduced in their areas in $1991,48(25.5 \%)$ in 1989 , three $(1.6 \%)$ in 1995 , two $(1.1 \%)$ in 1987 whilst the remaining $17(9.0 \%)$ did not answer this question.

With regard to the duration of services in the respective areas during the interview, of the 188 midwives, $12(6.4 \%)$ have been assigned in the area for less than a year, $61(32.4 \%)$ for $1-5$ years, $41(22.0 \%)$ for $6-10$ years, $23(12.2 \%)$ for $10-15$ years, and the remaining 51 (27.0\%) for 16 years and more.

Thirty-nine midwives (20.8\%) had no cases, $124(66.0 \%)$ had one to five cases, $15(7.9 \%)$ had 6-10 cases and three (1.6\%) interviewed midwives had more than 10 cases to be taken care of, whilst the remaining seven $(3.7 \%)$ did not mention their caseload. Thus, the average leprosy patient load was $2 \cdot 2$ cases per midwife per month. During the survey period, basic 
health services were provided by one midwife; an average of 15 patients per day. Thus, $2 \cdot 2$ leprosy patients count for only $0.75 \%$ of midwife's total caseload for a month.

All the interviewed midwives perceived that leprosy activities were not an extra workload. Ninety-four (50\%) of them were able to perform leprosy activities with other BHS activities. Seventy-three $(38.8 \%)$ presumed that it was a part of their routine activities under BHS since its integration with LEP ( $11 \%$ gave no answer).

Out of the 188 midwives interviewed, 149 (79\%) responded that they spent less than $10 \%$ and $18(10 \%)$ spent more than $10 \%$ of their monthly working time for LEP activities (11\% gave no answer).

Regarding the case finding activities, during the last 6-month period, the midwives conducted the routine contact survey, school survey and other methods (such as mass survey or cases detected during the physical examinations for other complaints). A total of 132 cases were suspected as leprosy by the interviewed midwives and 113 cases confirmed by their supervisors with an average accuracy rate of $85.6 \%$ (Table 1 ).

For contact survey, out of the 188 midwives interviewed, $147(78.2 \%)$ used this method and $41(21.8 \%)$ did not. Ninety-three cases were suspected as having leprosy. Of the 93 suspected cases, 80 were confirmed as having leprosy by their supervisors with the accuracy rate of $86.0 \%$.

For school survey, out of the 188 midwives interviewed, 147 (78.2\%) used this method, $40(21.3 \%)$ did not. (One gave no answer). Six cases were suspected as having leprosy. Of the six suspected cases, five were confirmed as having leprosy by their supervisors with an accuracy rate of $83.3 \%$.

For other surveys, out of the 188 midwives interviewed, $72(38.3 \%)$ used these methods and $102(54.2 \%)$ did not [14 $(7.5 \%)$ gave no answer]. Thirty-three cases were suspected as having leprosy. Of the 33 suspected cases, 28 were confirmed as having leprosy by their supervisors with the accuracy rate of $84.8 \%$.

A midwife spent $2 \cdot 1$ days during the last 6-month period for the surveys. The figure equals $1.9 \%$ of the total working days during half a year for each midwife.

In regards to the location of MDT drug delivery, $165(87 \cdot 8 \%)$ of the 188 interviewed midwives delivered MDT drugs in the patients' homes, seven $(3.7 \%)$ at the clinics (Health Centre/Sub-Centre), two $(1.1 \%)$ either in the homes or at the clinics, whilst the remaining 14 (7.4\%) were through other means, such as through the village health workers, or through another patient. It should be noted here that the distribution of MDT blister-packs containing 1 month's

Table 1. Leprosy cases detected by the interviewed midwives during last 6 months by different survey methods in the study areas of Myanmar in 1995

\begin{tabular}{lcccc}
\hline $\begin{array}{l}\text { Case detection } \\
\text { methods }\end{array}$ & $\begin{array}{c}\text { Midwives used } \\
\text { the method }^{\mathrm{a}}\end{array}$ & $\begin{array}{c}\text { Total number of } \\
\text { suspected cases }\end{array}$ & $\begin{array}{c}\text { Total number of } \\
\text { confirmed cases }\end{array}$ & $\begin{array}{r}\text { Percentage of case } \\
\text { detection accuracy }\end{array}$ \\
\hline $\begin{array}{l}\text { Contact survey } \\
\text { School survey }\end{array}$ & $147(78 \cdot 2 \%)$ & 93 & 80 & $86 \cdot 0 \%$ \\
$\begin{array}{l}\text { Other surveys } \\
\text { Total }\end{array}$ & $72(38 \cdot 3 \%)$ & 63 & 5 & $83 \cdot 3 \%$ \\
& & 132 & 113 & $84 \cdot 8 \%$ \\
\hline
\end{tabular}

\footnotetext{
${ }^{\text {a }}$ Some midwives used more than one case detection methods.

${ }^{\mathrm{b}}$ Other surveys include mass survey and case detection during the physical examinations for other complaints.
} 
drug was easier for the midwives to handle. They routinely collect the used blister-packs of the previous month from the patients whilst distributing the new supply of MDT drugs.

Periodic training was conducted for the midwives to implement the LEP activities. Out of the 188 interviewed midwives, $164(87.2 \%)$ responded that they had training whilst 24 $(12.8 \%)$ had no training on LEP activities by the time of the interview. With regard to the duration, of the 164 who received training, $26(15.9 \%)$ for 1 day, $30(18.3 \%)$ for 2 days, 103 $(62.8 \%)$ for 3 days, $3(1.8 \%)$ for more than 3 days [2 (1.2\%) gave no response].

With regard to any extra allowance for performing the LEP activities, out of the 188 interviewed midwives, $182(96.8 \%)$ responded did not receive any extra allowance, three (1.6\%) responded received occasional extra allowance. The remaining three $(1.6 \%)$ did not mention either.

The midwives were required to submit a monthly report to their field supervisors. To prepare such a report, they kept records of all activities that they carried out. Out of the 188 interviewed midwives, $177(94.2 \%)$ responded that they maintained a separate stock book, patient charts and records of leprosy cases. Ten (5.3\%) did not keep such records and one $(0.5 \%)$ did not respond either.

All the interviewed midwives responded that their LEP activities were supervised by both LEP and BHS staff at the different levels. In all, $81.4 \%$ of the total supervisory visits were by the BHS staff and the remaining $18.4 \%$ by the LEP staff.

\section{Discussion}

The MDT implementation guidelines of WHO simplified the LEP activities such as case detection and drug distribution in the endemic countries. LEP activities were distributed to the BHS staff through an integrated approach to implement the MDT. This study revealed that an average of 2.2 leprosy patients count only $0.75 \%$ of a midwife's total monthly workload among those interviewed. Moreover, all midwives perceived that leprosy activities were not an extra burden, as $94(50 \%)$ of them were able to perform these activities with other BHS activities and $73(38.8 \%)$ presumed that it was a part of their routine activities under BHS since its integration with LEP. Thus, LEP is implemented by the BHS midwives without hampering other basic health services of the country and without any extra allowance for the LEP activities. Training on LEP activities was one of the essential task which is strengthened by regular supervisory visits and on the job training of the midwives.

Along with the present trend of decreasing prevalence rate and maintenance of $100 \%$ of MDT coverage over the registered cases, it is evident that leprosy will not be a significant public health problem at the national level by the year 2000 in Myanmar. However, because of the long incubation period of the disease, new leprosy patients may present long after the elimination target is achieved, whilst many other patients may develop disability. Thus, a community-based, sustainable approach for the post-elimination phase, after the year 2000, will be essential and the contribution of the midwives may be of considerable importance.

\section{Acknowledgement}

Special acknowledgement is extended to Dr A. Colin McDougall, former Editor of Leprosy Review, for his valuable comments to finalize this manuscript. One of us wishes to extend 
his gratitude to the Sasakawa Memorial Health Foundation (SMHF), Tokyo, Japan for support in collecting data for this study whilst monitoring the Leprosy Elimination Program of Myanmar on behalf of the SMHF during 1995-1997. Kind permission of the Ministry of Health $(\mathrm{MOH})$ of the Myanmar enabled us to conduct the study. We wish to record our thanks to the staff of the MOH even at the Health Center and Health Sub-Center levels without whose support and cooperation the study would have been impossible.

\section{References}

1 WHO. Chemotherapy of leprosy for control programmes. Report of a WHO Study Group, Geneva (WHO Technical Report Series, No. 675), 1982.

2 WHO. Action program for the elimination of leprosy. Status Report, Geneva, 1996.

3 WHO. A guide to eliminating leprosy as a public health problem. Geneva, General (E), WHO/LEP/95. 1995; 1: $5-6$.

4 Yuasa Y. MDT for all: target oriented leprosy control program in 1990's. Int J Lep Other Mycobact Dis, 1991; 57: 624-638.

5 WHO. Action programme for the elimination of leprosy. Status Report, Geneva, 1998.

6 WHO. Weekly Epidemiol Rec, 1997; 72: 165-172.

7 Kyaw Lwin, Zuiderhoek B. Leprosy control in Myanmar (Burma): a retrospective view of the tackling of a huge leprosy problem and its results over a 25-year period (1948-1973). Working Group on History, Netherlands Society of Tropical Medicine, 1997. 